\title{
Factores que inciden en la prueba de competencias científicas en estudiantes colombianos
}

\section{Factors that affect the test of scientific competencies in Colombian students}

\author{
VELASCO, Andrés ${ }^{1}$ \\ BARRIOS, Nelson ${ }^{2}$ \\ PALACIOS, Jairo ${ }^{3}$
}

\begin{abstract}
Resumen
El estudio de los factores asociados al desarrollo de competencias científicas en estudiantes de primaria de las instituciones educativas oficiales se realiza mediante un análisis estadístico de significancia bivariada que permite observar el nivel de asociación entre las categorías utilizadas. Para esta investigación se diseña y aplican tres instrumentos: dos a estudiantes (prueba de competencias científicas y factores asociados) y uno a docentes (factores asociados). Los resultados enfocan la atención del aprendizaje en aspectos propios del contexto, enseñanza de las ciencias, escuela y familia, resaltando la influencia de estos en la apropiación de las competencias científicas.

Palabras clave: ambiente educacional, aprendizaje, conocimiento científico.
\end{abstract}

\begin{abstract}
The behavior of factors associated with the development of scientific competencies in elementary students of official educational institutions, a statistical analysis of bivariate significance is performed to observe the level of association, three instruments are designed and applied: two to students (test of scientific competences and associated factors) and one to teachers (associated factors), the results focus attention on learning aspects of the context, science teaching, school and family.

key words: educational environment, learning, scientific knowledge.
\end{abstract}

\section{Introducción}

Según Coronado \& Arteta (2015) las competencias científicas se definen como el conjunto de conocimientos, capacidades y actitudes que permiten a los estudiantes actuar en diferentes contextos donde se requiera de la aplicación de conceptos científicos. Estas competencias son evaluadas por organismos nacionales e internacionales, en Colombia el Instituto Colombiano para el Fomento de la Educación Superior (Icfes) evalúa a los niveles de 3우 , 5으, 9으, 11으 y PRO en el marco de la prueba Saber y, a nivel internacional, la Organización para la Cooperación y el Desarrollo Económico (OCDE) evalúa a estudiantes de 15 años. Asímismo, el Laboratorio Latinoamericano de Evaluación de la Calidad de la Educación (LLECE) evalúa los niveles de tercero de sexto grado

\footnotetext{
${ }^{1}$ Docente. Facultad de Facultad de Ciencias Humanas. Escuela de educación. Universidad Industrial de Santander. Doctor(e) en educación andresvc556@hotmail.com. ORCID: 0000-0002-5713-6334.

${ }^{2}$ Docente. Facultad de Medio Ambiente y Recursos Naturales. Programa de Saneamiento Ambiental y recursos naturales. Universidad Distrital Francisco José de Caldas. Doctor en Educación. nebarriosj@udistrital.edu.co, ORCID: 0000-0003-0815-201X.

3 Docente. Facultad de Ciencias Sociales. Programa de Trabajo Social. Universidad Colegio Mayor de Cundinamarca. Magister en Educación jjpalacios@unicolmayor.edu.co ORCID: 0000-0002-1437-9838.
} 
respectivamente. De tal manera, estas pruebas permiten generar resultados para identificar el estado actual de las capacidades de los estudiantes y hacer seguimiento a su desarrollo, así mismo, posibilitan la formulación de planes de mejoramiento y actividades pedagógicas que tienen en cuenta las debilidades y fortalezas de los estudiantes y permitan mejorar su desempeño académico (Icfes, 2016), igualmente, a nivel internacional ayudan a hacer sinergia regional hacia la mejora de los aprendizajes.

En concordancia, los resultados son estudiados a la luz de los factores asociados. En palabras de Barahona, Veres, \& Barahona (2018) el éxito académico de los estudiantes es multifactorial, es decir, tiene que ver con determinantes personales, sociales e institucionales, por ello, las pruebas SABER, PISA y TERCE presentan un cuestionario sociodemográfico que permite reconocer la situación escolar, social y económica que atraviesan quienes presentan dichas pruebas, sin embargo, en muchas ocasiones los resultados se presentan de forma general y no tienen una relación directa de dichos resultados en un contexto, área y zona específica.

Por tal razón, este artículo presenta una medición del nivel de competencias científicas en los estudiantes de quinto de primaria de las instituciones oficiales del municipio de Bucaramanga. Así mismo, se aplica un cuestionario de factores asociados a los estudiantes y docentes de cada grado, buscando con ello realizar un contraste entre los resultados que genere el nivel de asociación entre los factores y los resultados obtenidos en la prueba de competencias científicas.

Al finalizar quinto grado los estudiantes están concluyendo su educación primaria, en este nivel académico se presenta la prueba Saber 5 o, sin embargo, no siempre se incluye el cuestionario de competencias científicas, para el caso de Bucaramanga esta prueba se ha aplicado desde el año 2009 y a la fecha se han presentado en 7 años diferentes, el cuestionario en competencias científicas se ha incluido solo en 3 ocasiones y en el año 2016 no se incluyeron las tres competencias a evaluar que comprenden las competencias científicas. Esta situación deja un vacío en la realidad acerca del desarrollo de este tipo de competencias en niños y niñas de quinto grado de las instituciones de la ciudad.

Por otro lado, el cambio de primaria a secundaria generalmente es traumático y genera deserción en los estudiantes. Situaciones como el paso de un solo docente que enseña todas las asignaturas a contar con docentes especializados para cada asignatura en secundaria, la variabilidad en el enfoque de las clases y cambios conceptuales en los conocimientos adquiridos y las características en el aula evidencian un contexto diferente en los estudiantes de sexto grado. Por tal razón, desde el área de ciencias naturales se puede dar un paso importante en la enseñanza de las ciencias y el acercamiento de los estudiantes gracias a la comprensión de las competencias científicas, para ello es fundamental identificar su estado actual, los factores o elementos que influyen en el desarrollo de las mismas.

Las características mencionadas en el párrafo anterior, permiten plantear el trabajo descrito mediante la identificación de los factores que inciden en el proceso de enseñanza y aprendizaje de las ciencias naturales, los cuales enmarcan las distintas realidades de los estudiantes, no como clasificación general, sino como un reconocimiento de las características de los niños y niñas del municipio. De esta manera, se construyeron tres cuestionarios: primero, una prueba de competencias científicas con 24 preguntas liberadas por el Icfes que incluyen los niveles de desempeño, competencias y componentes. Segundo, un cuestionario sociodemográfico a estudiantes compuesto por 37 preguntas y tercero, un cuestionario a docentes con 13 preguntas. Estos dos últimos fueron diseñados con relación a los cuestionarios sociodemográficos de las pruebas Saber, PISA y TERCE.

Para efectos del análisis, se utilizó el software libre R (R Core Team, 2018) el cual ofrece variedad de funciones en el momento de acceder y gestionar los datos, así como analizarlos y representarlos. De esta manera, se procedió en tres momentos: 
Primero, análisis exploratorio univariado de los tres instrumentos, donde se usó descripción por medio de frecuencias relativas. Segundo, análisis de significancia bivariado de los resultados de la prueba de competencias con relación a los factores asociados, para lo cual se empleó la prueba de Chi-cuadrado ( $\chi 2$ ) para conocer el grado de asociación individual de las variables, y, tercero, se construyó un modelo de regresión logística multinominal o multivariante con el fin de identificar las probabilidades de registro de los resultados o desempeño.

A partir del análisis se identificó, en primer lugar, la presencia de diferencias en los resultados de las pruebas de competencias científicas de las instituciones, específicamente en la media y la desviación estándar, dando a docentes e instituciones un valor agregado en la adquisición del conocimiento gracias a los procesos de enseñanza-aprendizaje.

Segundo, se evidenciaron aspectos positivos, negativos y preocupantes en los cuestionarios aplicados a los estudiantes, así como en los aplicados a los docentes. Estas situaciones son de profundo análisis, por cuanto abren las posibilidades a nuevas investigaciones que integren a los estudiantes y los factores asociados al desarrollo de competencias científicas.

Tercero, se determinaron aspectos relacionados con los resultados de la prueba de competencias científicas. El estudio permitió reconocer que estos resultados no son parte del azar; así mismo, la prueba de chi cuadrado demostró que existe una asociación significativa entre los desempeños y los factores asociados, por ello se concluye que es posible mejorar en la prueba de competencias científicas si se genera influencia en algunos factores asociados estudiados en los cuestionarios aplicados.

No obstante, al realizar el Análisis de Componentes Principales (ACP), no se encuentró claridad en la relación entre factores asociados y puntajes obtenidos de acuerdo a la cercanía existente en el plano producto de la investigación. En este sentido, desde la Unesco (2015) se resaltan algunos aspectos fundamentales: las características de los estudiantes y sus familias tienen una robusta asociación con los logros de aprendizaje, en razón a lo anterior, el apoyo parental es fundamental para el desempeño y el progreso académico de niñas y niños.

Asímismo, se determinó que el índice de nivel socioeconómico es tomado como un índice que ubica en diferentes niveles el aprendizaje de los estudiantes en todos los países, disciplinas y grados, haciendo referencia a la tenencia de ciertos artículos importantes en los procesos formativos, por ejemplo, la presencia de computador en la casa. El docente y las prácticas en el aula se convierten en las principales variables que afectan el rendimiento escolar, de esta manera, vale la pena resaltar que la formación y capacitación docente permite la creación de espacios más agradables en el proceso de enseñanza y aprendizaje de las ciencias.

Los anteriores elementos se encuentran en concordancia con el estudio realizado y permite comprender las puertas que se abren a corto, mediano y largo plazo. A corto plazo se manifiesta una reflexión institucional acerca de los procesos que se llevan en el aula y la integración de los factores que se asocian al aprendizaje de las ciencias. A mediano plazo, se resalta el diseño y aplicación de estrategias que posibiliten el mejoramiento de los procesos de enseñanza y aprendizaje en el aula, y a largo plazo, el análisis secuencial de los resultados con el fin de controlar y puntualizar en las variables que influyan en los resultados de las pruebas de competencias científicas en los diferentes niveles académicos.

\section{Metodología}

La investigación se desarrolla bajo un enfoque Cuantitativo, como lo señala Hernández-Sampieri (2014) estos estudios se orientan a explorar conceptos o relacionarlos con el fin de medir unas variables y generar asociación. Por tal razón, el propósito de esta investigación consiste en identificar y relacionar los factores que se asocian a 
los resultados de competencias científicas en estudiantes de quinto grado del municipio de BucaramangaSantander. Teniendo en cuenta los requerimientos del estudio realizado se toma como población los estudiantes y docentes de quinto grado de las 48 instituciones educativas oficiales del municipio de Bucaramanga-Santander. Por lo tanto, la muestra corresponde a 25 grados de quinto primaria con sus docentes correspondientes a diferentes instituciones educativas oficiales descritos en la tabla 1.

Tabla 1

Descripción de la Muestra (estudiantes)

\begin{tabular}{|c|c|c|}
\hline Características & Categorías & Porcentaje (\%) \\
\hline Sexo & Femenino & $48.43 \%$ \\
& Masculino & $51.32 \%$ \\
\hline \multirow{2}{*}{ Edad } & No identificado & $0.25 \%$ \\
\hline & $9-10$ & $62.61 \%$ \\
\hline & $11-12$ & $33.75 \%$ \\
\hline & $13-15$ & $3.39 \%$ \\
\hline & No identificado \\
señalada en el cuestionario. & $0.25 \%$ \\
\hline
\end{tabular}

Fuente: elaboración propia

La recolección de la información mediante la encuesta como instrumento de investigación permite eliminar el contacto cara a cara con el encuestado, sin embargo, la construcción de los cuestionarios requiere de rigor y calidad lingüística para captar la totalidad de la información que se requiere sin distorsionarla. En este sentido, Vivanco (2005) señala que una de las principales bondades de la encuesta es poder utilizar la información de una muestra representativa para explorar, describir o explicar la población origen. Así mismo, el cuestionario corresponde al instrumento utilizado por los investigadores para hacer realidad la encuesta, para cumplir con el objetivo planteado en el presente trabajo de investigación se han diseñado tres cuestionarios necesarios para recolectar la información lo más completa posible. Estos se relacionan en la tabla 2.

Tabla 2

Descripción de los Cuestionarios

\begin{tabular}{|c|c|c|}
\hline Cuestionario & Encuestado & \# de preguntas \\
\hline Competencias científicas & Estudiantes & 24 \\
\hline Factores asociados & Estudiantes & 37 \\
\hline Factores asociados & Docentes & 13 \\
\hline
\end{tabular}

Fuente: elaboración propia

Para el desarrollo del presente estudio se plantearon las siguientes etapas:

Primera etapa: Diseño de los instrumentos, consiste en la construcción de los instrumentos para obtener la información requerida para alcanzar el objeto propuesto. De esta manera, se construyeron teniendo en cuenta la revisión bibliográfica y el análisis de pruebas internacionales, se construyeron de la siguiente manera:

- Cuestionario de competencias científicas: se realizó la revisión de las pruebas Saber 5o aplicadas desde el año 2009 y se utilizaron las preguntas liberadas por el Icfes para fines investigativos. Para cumplir adecuadamente se tuvo en cuenta las competencias que se evalúan en la prueba, a saber, uso comprensivo del conocimiento científico, explicación de fenómenos e indagación y los componentes que configuran estas competencias los cuales son: entorno vivo, entorno físico y ciencia, tecnología y sociedad.

- Factores asociados: los cuestionarios 2 y 3 correspondientes a factores asociados a estudiantes y docentes se construyeron teniendo en cuenta los aspectos sociodemográficos de las pruebas Saber, Pisa y Terce. Es 
decir, se tomaron los cuestionarios, a partir de ello se construyeron las macrocategorías foco de esta investigación. A saber, familia, contexto, escuela y enseñanza de las ciencias.

Segunda etapa: aplicación de los instrumentos, se elige la muestra a conveniencia teniendo en cuenta que el universo de la población cumple con los mismos criterios acordes a la naturaleza de la investigación, por consiguiente, previa autorización de la secretaria de educación municipal y en común acuerdo con rectores, coordinares y docentes se aplicaron los cuestionarios a la muestra.

Tercera etapa: análisis de la información, después de aplicados los cuestionarios se procede a tabular la información de forma organizada y codificada, con el fin de tenerla lista para el análisis estadístico planteado en el siguiente título y la respectiva discusión de los resultados.

Para el desarrollo del análisis estadístico fue necesario emplear varios procedimientos, descritos a continuación:

1. Análisis exploratorio univariado de los instrumentos, se realizó mediante frecuencias relativas donde son presentadas las respuestas a manera de porcentajes (\%) de esta forma el análisis es favorable para la interpretación de la información.

2. Análisis de significancia bivariado de la variable dependiente frente a las variables independientes, por tal razón, se realiza un análisis de tablas de contingencia buscando identificar el nivel de asociación entre la variable dependiente reconocida como factores y la variable independiente "resultados de la prueba de competencias científicas" para cada una de las categorías, para la variable dependiente se utilizaron los cuestionarios 2 y 3 de factores asociados enunciados en la Tabla 2 y para la variable independiente el cuestionario 1, dada la necesidad de una escala cualitativa para el cuestionario 1 los resultados se modificaron teniendo en cuenta cuatro rangos de desempeño. (Ver tabla 4).

Tabla 3

Listado de variables de investigación

\begin{tabular}{|l|l|ll|}
\hline \multicolumn{1}{|c|}{ Tipo } & \multicolumn{1}{|c|}{ Variable } & & \multicolumn{1}{c|}{ Indicadores } \\
\hline Dependiente & Factores en el & a. & Familia \\
& proceso de & b. Contexto socioeconómico \\
& enseñanza & $\begin{array}{l}\text { c. Enseñanza de las Ciencias } \\
\text { d. Escuela }\end{array}$ \\
\hline Independiente & $\begin{array}{l}\text { Competencias } \\
\text { científicas }\end{array}$ & $\begin{array}{l}\text { a. Uso comprensivo del conocimiento científico } \\
\text { b. Explicación de Fenómenos }\end{array}$ \\
& & c. Indagación \\
\hline
\end{tabular}

Fuente: elaboración propia

Tabla 4

Rangos de desempeño de resultados

\begin{tabular}{|c|c|c|c|}
\hline \multicolumn{3}{|c|}{ Escala cualitativa } & Escala cuantitativa \\
\hline 43.6 & - & 48 & Desempeño superior \\
\hline 38.6 & - & 43.5 & Desempeño alto \\
\hline 27.6 & - & 38.5 & Desempeño básico \\
\hline 0 & - & 27.5 & Desempeño bajo \\
\hline
\end{tabular}

Fuente: elaboración propia

1. Buscando conocer el grado de asociación individual de las variables se usó la prueba de Chi-cuadrado ( $\chi 2)$, de acuerdo con el resultado, cuando el nivel de significación es menor ó igual a 0.05 se rechaza la hipótesis, por ello, se rechazó la hipótesis de independencia, corroborando la asociación de dichas variables. Para concluir este análisis, se busca observar la fuerza de la asociación a través del estadístico 
V de Cramer, este oscila entre 0 y 1 (valores cercanos a 0 indican no asociación y los próximos a 1 fuerte asociación) (Pardo \& Ruiz, 2001).

2. Para finalizar, los factores que mostraron una asociación fuerte se incluyeron en un modelo de regresión logística multinominal o multivariante, identificando las probabilidades de registro de los resultados o desempeño. Así, se valoraron varios factores simultáneamente estuvieran presumiblemente relacionados, según resultados de Chi-cuadrado $(\chi 2)$, con la variable dependiente "resultados o desempeño", identificando su efecto de forma ajustada (exponencial de B).

Esta investigación con enfoque cuantitativo, busca indagar sobre aspectos generales del proceso de enseñanza y aprendizaje de las ciencias naturales, específicamente en el comportamiento de los resultados en la prueba de competencias científicas, con el propósito de buscar alternativas para mejorar los procesos académicos, los cuales se enuncian a continuación:

1. Para la aplicación de los instrumentos se contó con la previa autorización de la secretaria de educación municipal y el permiso de ingreso a las instituciones de rectores o coordinadores.

2. Siempre y de manera estricta se protege la propiedad intelectual de las fuentes citadas o referenciadas en el documento.

3. No se hará uso de datos personales garantizándose la privacidad y confidencialidad respecto a los datos institucionales y/o de los sujetos participantes.

4. Desde los datos recolectados se socializarán salvaguardando la información y datos recogidos de instituciones o personas. En caso de utilizarse uso abierto de los datos para Crowdsourcing Research, los datos y consideraciones tendrán absoluta seguridad informática y principio de confidencialidad.

\section{Resultados}

Para mantener un orden adecuado en la presentación y explicación de los resultados, se reportarán los resultados por etapas:

Primera etapa: Se realizó el análisis exploratorio univariado que describe los resultados de las pruebas de competencias científicas y se presentan los resultados de los cuestionarios de factores asociados, identificando los porcentajes de respuestas elevados para cada categoría.

Segunda etapa: Se hizo el análisis de significación bivariado. Esta etapa cobró valor porque identificó los factores que tienen asociación los resultados de la prueba de competencias científicas mediante la prueba de ChiCuadrado teniendo como referente de significación.

Tercera etapa: durante esta etapa se dieron a conocer los factores que mostraron asociación y fuerza a través del estadístico $V$ de Cramer, a partir de ello, se diseñaron los modelos dando como resultado la probabilidad de obtener mejores resultados en mayor número de veces.

Cuarta etapa: para identificar la asociación general de puntaje y los factores, se realizó un análisis de componentes principales (ACP) con los factores que mostraron probabilidad de mejorar los resultados de la prueba. 


\section{Primera etapa: Análisis exploratorio univariado}

El cuestionario 1 denominado "prueba de competencias científicas" se aplicó en 25 grados quintos de básica primaria de diferentes instituciones educativas.

Gráfica 1

Resultados de la prueba de Competencias Científicas

90

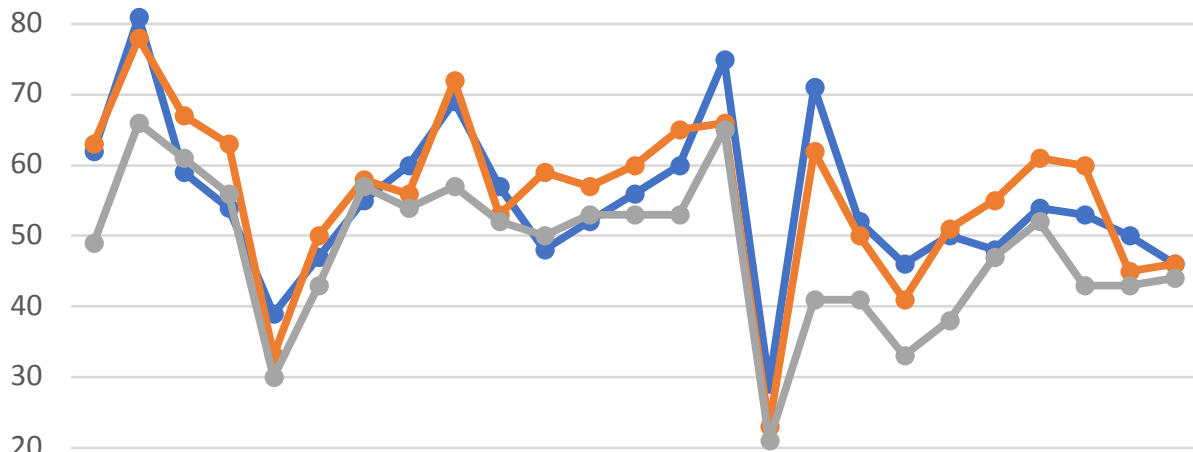

10

0

\begin{tabular}{|l|l|l|l|l|l|l|l|l|l|l|l|l|l|l|l|l|l|l|l|l|l|l|l|l|}
\hline 1 & 2 & 3 & 4 & 5 & 6 & 7 & 8 & 9 & 10 & 11 & 12 & 13 & 14 & 15 & 16 & 17 & 18 & 19 & 20 & 21 & 22 & 23 & 24 & 25 \\
\hline
\end{tabular}

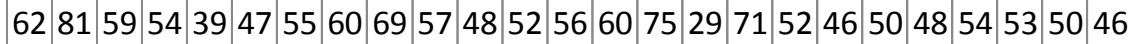

Uso del Conocimiento
- Explicación de Fenómeno

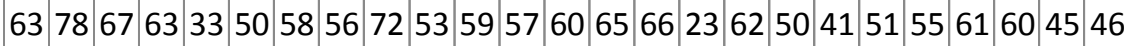

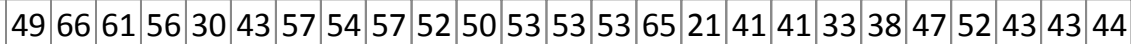

Instituciones

Fuente: Elaboración propia

En la gráfica 1 se observan los resultados de las 25 instituciones, en cada una de las tres competencias que evalúa el Icfes, en ella se evidencia la siguiente información:

La institución con mayor puntaje promedio corresponde a la №2, siendo una institución donde la capacitación docente, el orden de clase y la planeación son evidentes gracias a los trabajos que se realizan a nivel institucional. En contraposición, la institución con menor puntaje corresponde a la № 16, donde se evidencia desorden en el aula y poco manejo de la planeación en el área de ciencias naturales, esta situación común en muchas aulas de quinto se da cuando el docente titular del grado es especialista en otra asignatura.

De esta manera, se evidencia que no mantiene un equilibrio entre los resultados de las diferentes instituciones educativas, por tal razón, existen variables que junto con el proceso de enseñanza y aprendizaje tienden a influir en la obtención de mejores resultados. La competencia que se destacó en la mayoría de las instituciones es el uso comprensivo del conocimiento científico, seguida de la competencia Explicación de fenómenos con puntajes muy próximos, por otro lado, la competencia Indagación mostro resultados bajos en la mayoría de las instituciones, razón que manifiesta que las competencias científicas se desarrollan de forma secuencial, inicialmente el uso del conocimiento que permite generar explicaciones frente a los diversos fenómenos observados en la naturaleza, dando paso a la indagación frente a los mismos fenómenos. 
Gráfica 2

Porcentaje (\%) con desempeño

bajo en cada competencia evaluada

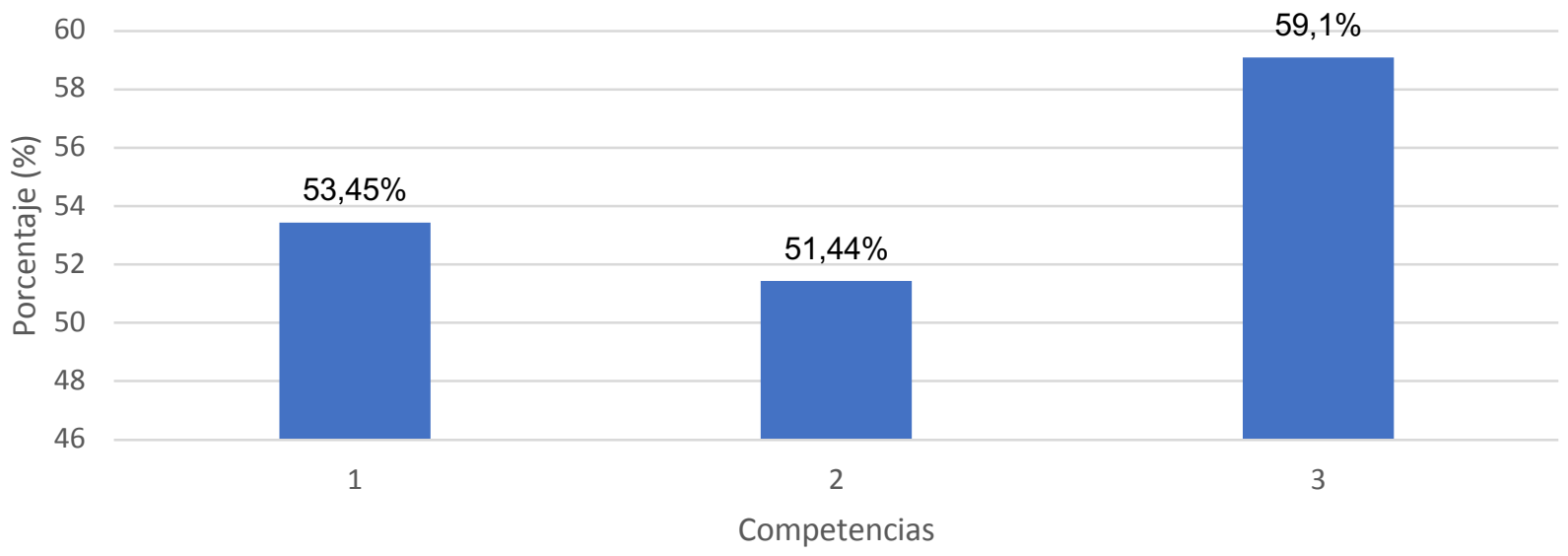

Fuente: Elaboración propia

En relación al ítem anterior, la gráfica 2 muestra los porcentajes (\%) en general con desempeño bajo de las tres competencias evaluadas, se puede observar que la competencia indagación (3) obtiene 59,1\% de los estudiantes en desempeño bajo. Por otro lado, se reconoce un mayor porcentaje (\%) de estudiantes con desempeño bajo en la competencia uso del conocimiento frente a Explicación de Fenómenos, en contraposición a lo observado en la gráfica N1 donde los resultados de la primera competencia son más altos.

Gráfica 3

Porcentaje por desempeño a nivel general de la prueba de Competencias Científicas

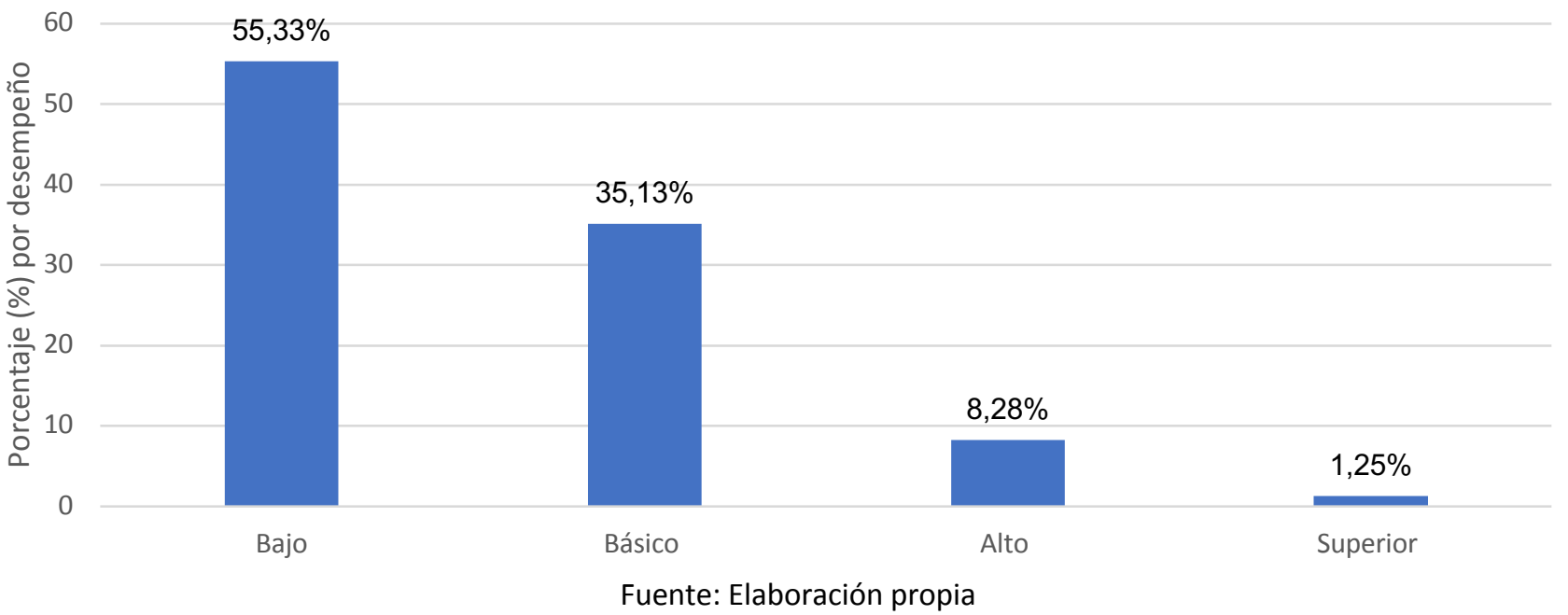

Así mismo, la gráfica 3 da a conocer los resultados por desempeño de la prueba. Evidentemente, el 90,46\% de estos se encuentran ubicados entre los desempeños bajo y básico. Esta situación preocupante permite profundizar en el análisis de los factores que influyen en los resultados de la prueba y generan el interés para buscar alternativas que posibiliten la obtención de mejores resultados.

Los cuestionarios dos y tres denominados "factores asociados" permiten identificar aspectos generales que generan ruido frente a las macrocategorías dispuestas para el estudio. De esta manera, los factores indagados por cada categoría están descritos en una tabla y categorizados en una gráfica de barras con diferentes colores 
donde el azul señala un aspecto positivo, el naranja un aspecto negativo y el morado un aspecto preocupante, aspectos que se evidencian en las siguientes categorías.

En la tabla 5 se relacionan los factores indagados de la categoría familia, la gráfica N 4 señala como aspectos positivos que los estudiantes asisten constantemente a clase, dedican suficiente tiempo para el desarrollo de las actividades en el hogar y se tiene amplia expectativa frente al nivel académico que se alcanzara, por ello, se cuentan con espacios en el hogar para realizar las actividades y sus padres proporcionan los materiales de trabajo de forma oportuna.

Tabla 5

Factores relacionados a la Categoría Familia

\begin{tabular}{|c|l|}
\hline \multicolumn{2}{|c|}{ Categoría Familia } \\
\hline 1 & \multicolumn{1}{|c|}{ Factor } \\
\hline 2 & Asistencia a clase. \\
\hline 3 & Repitencia de grados en básica primaria. \\
\hline 4 & Retiro del colegio sin justificación. \\
\hline 5 & Dedicación en el desarrollo de tareas en el hogar. \\
\hline 6 & Dedicación desde el hogar para el estudio. \\
\hline 7 & Falta de supervisión en actividades por parte de los padres de familia. \\
\hline 8 & Espacio adecuado para desarrollar tareas en el hogar. \\
\hline 9 & Compra de textos cuando se necesitan. \\
\hline 10 & Preocupación de los padres por el desempeño de los estudiantes. \\
\hline 11 & Preocupación por el mejoramiento académico. \\
\hline 12 & Expectativas sobre el nivel académico que alcanzarán los estudiantes. \\
\hline &
\end{tabular}

Sin embargo, se tienen aspectos negativos o por mejorar:

Primero, la falta de justificaciones cuando se ausenta de clase es un común denominador, llevando consigo una gran responsabilidad por parte de los padres de familia, quizás por falta de compromiso.

Segundo, muchos estudiantes manifiestan que se han retirado del colegio sin justificación.

Tercero, es común el hecho de repetir grado en básica primaria, situación que genera descompensación en la edad adecuada para llegar al bachillerato, dando como resultado la deserción escolar. 
Gráfica 4

Resultado en porcentaje de los

factores de la Categoría Familia

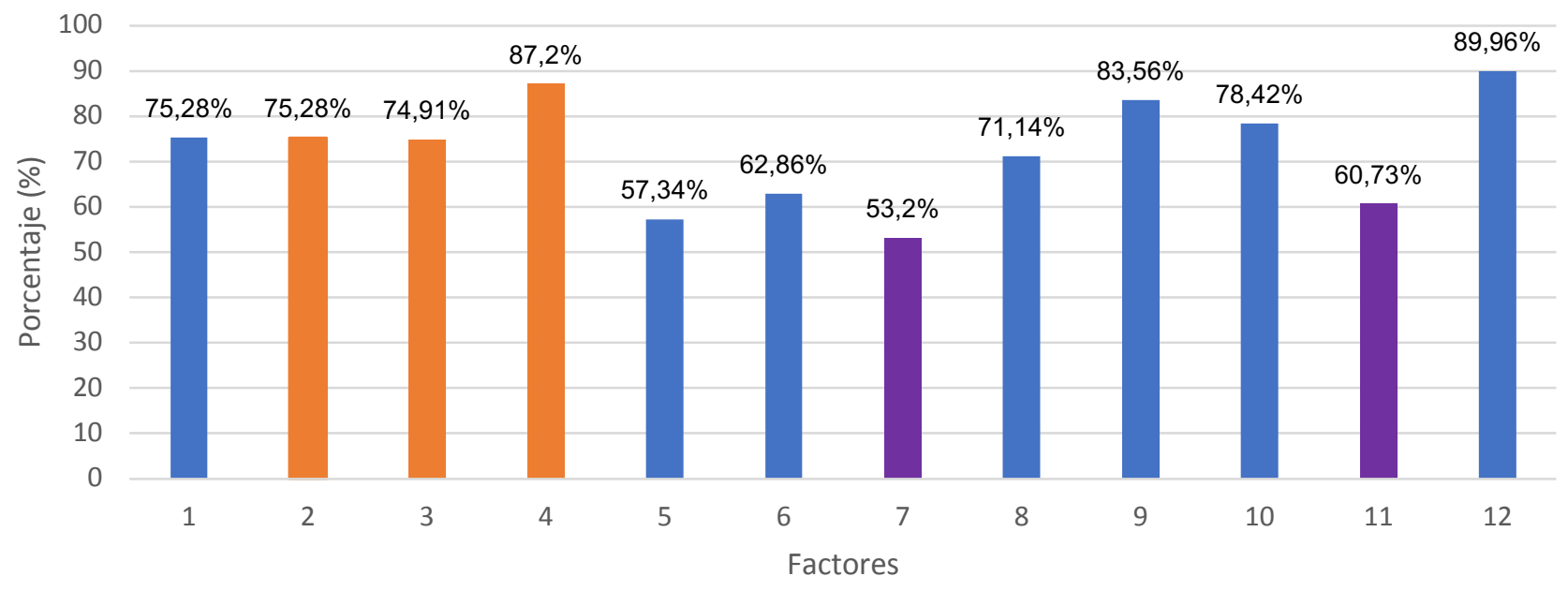

Fuente: Elaboración propia

En la tabla 6 se relacionan los factores asociados a la categoría contexto socioeconómico, después de la lectura y tabulación de los resultados se ha encontrado que todos los aspectos de esta categoría se pueden designar como negativos, es decir, que afectan el proceso de enseñanza y aprendizaje en los estudiantes. Teniendo en cuenta que la población objeto de estudio se centra en los estratos $0,1,2$ y 3 en el mejor de los casos, las situaciones que viven los estudiantes a diario hacen del contexto un factor influyente en su futuro.

Situaciones como el rechazo y la violencia en el hogar, junto con la violencia del entorno generan mal ambiente en el momento de estudiar, así mismo, la ausencia de uno de los padres, la inestabilidad laboral tanto de padres como de madres y la falta de formación académica no se consideran el mejor ejemplo para niños y niñas. Sin embargo, se debe destacar que, aunque la formación académica de los padres no es la esperada, en la categoría familia se identificó el interés de los padres por el mejoramiento académico de sus hijos como una oportunidad para mejorar su nivel socioeconómico.

Tabla 6

Factores relacionados a la Categoría Contexto Socioeconómico

\begin{tabular}{|c|l|}
\hline No & \multicolumn{1}{|c|}{ Factor } \\
\hline 1 & Incremento del trabajo infantil remunerado. \\
\hline 2 & Altas tasas de violencia en el entorno del hogar. \\
\hline 3 & Altas tasas de violencia en el hogar. \\
\hline 4 & Rechazo en el hogar. \\
\hline 5 & Desigualdad en los ingresos económicos y bienes que poseen en el hogar. \\
\hline 6 & Falta de oportunidades para el acceso a actividades extracurriculares. \\
\hline 7 & Falta de familias convencionales en los hogares (ausencia de padre). \\
\hline 8 & Falta de familias convencionales en los hogares (ausencia de madre) \\
\hline 9 & Elevada tasa de analfabetismo de primaria en los adultos del hogar. \\
\hline 10 & Falta de educación secundaria en los adultos del hogar. \\
\hline 11 & Falta de educación universitaria en los adultos del hogar. \\
\hline 12 & Inestabilidad laboral de los padres de familia (padre). \\
\hline 13 & Inestabilidad laboral de los padres de familia (madre). \\
\hline
\end{tabular}

Fuente: Elaboración propia 
Gráfica 5

Resultado en porcentaje de los factores

de la Categoría Contexto Socioeconómico

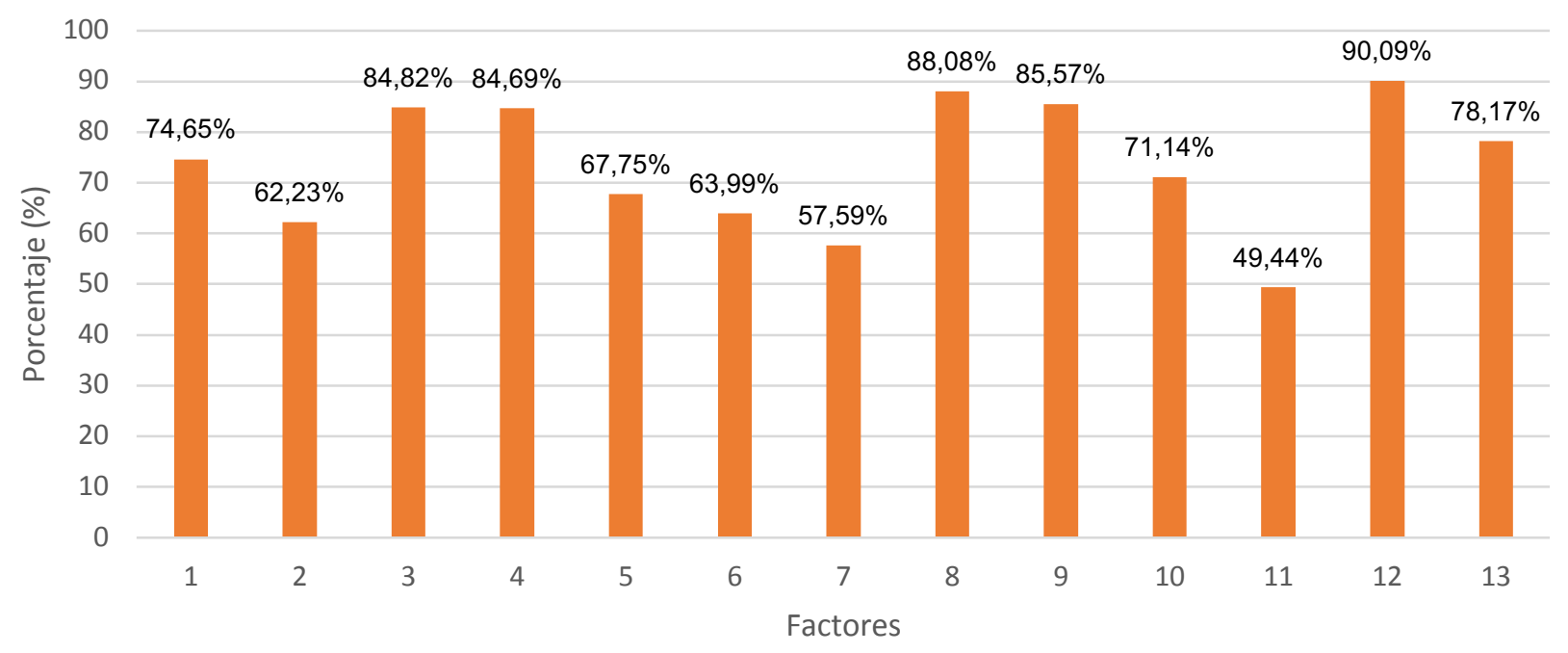

Fuente: Elaboración propia

En la tabla 7 se relacionan los factores que conforman la categoría Enseñanza de las Ciencias, como se observa en la gráfica N 6, se cuentan con aspectos positivos y negativos, en el grupo de los positivos se observa que a los estudiantes de quinto primaria les gusta la clase de ciencias naturales y mantienen el interés por el estudio de la misma, factores fundamentales en el momento de iniciar un proceso de aprendizaje. De la misma manera, se destaca la percepción que tienen los estudiantes sobre los docentes, manifestando que se desarrollan clase dinámicas y le dan importancia a las tareas que se dejan en clase, además, los docentes realizan las respectivas correcciones a tareas y evaluaciones. En contraposición, aunque se manifestó que existe dinamismo en la clase de ciencias naturales, no hay uso del laboratorio o la sala de informática para la enseñanza de las ciencias, situación que dificulta el desarrollo de competencias científicas. Así como, emplear estas tecnologías en la formación de los recursos humanos necesarios para la era de la globalización (Clavijo, 2018).

Tabla 7

Factores relacionados a la Categoría

Enseñanza de las Ciencias

\begin{tabular}{|c|l|}
\hline N & \multicolumn{1}{|c|}{ Factor } \\
\hline 1 & Gusto por las ciencias. \\
\hline 2 & Interés por la clase de ciencias. \\
\hline 3 & Identificación de clases monótonas \\
\hline 4 & Poco o ningún uso del laboratorio para los temas de clase. \\
\hline 5 & Desinterés por las TIC en la clase. \\
\hline 6 & Constante indisciplina en clase. \\
\hline 7 & Importancia en las tareas dejadas en clase. \\
\hline 8 & Correcciones por parte del docente en clase \\
\hline 9 & Correcciones por parte del docente a evaluaciones \\
\hline 10 & Dinamismo de actividades de clase \\
\hline
\end{tabular}

Fuente: Elaboración propia 


\section{Gráfica 6}

Resultado en porcentaje (\%) de los factores de la Categoría Enseñanza de las Ciencias

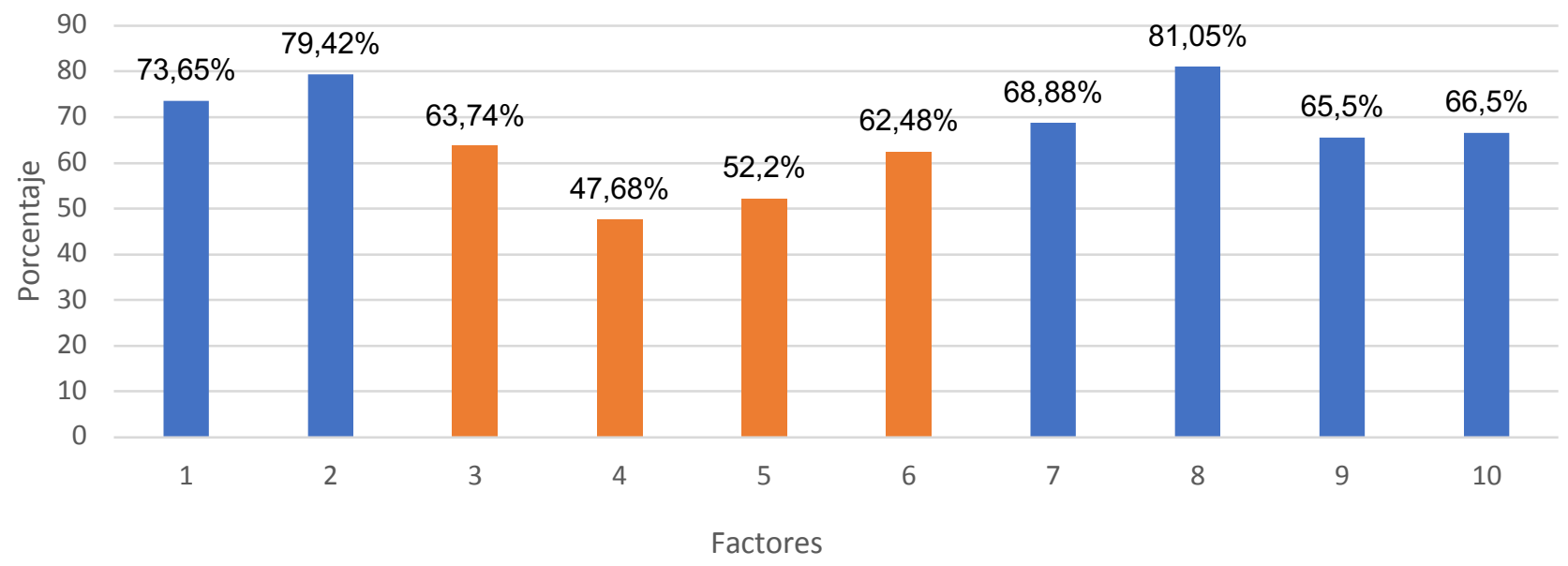

Fuente: Elaboración propia

La categoría escuela se toma del cuestionario 3 aplicado a los docentes, en la gráfica 7 muestra que para los docentes no existen diferencias entre lo que ellos enseñan con relación a grupos de su misma institución o de otras. Además, se señala que ni la influencia del contexto, ni la ausencia de útiles son impedimento para que los estudiantes desarrollen aprendizajes. Así mismo, manifiestan que los estudiantes tienen acceso a elementos de tecnología fuera de la institución y que no es necesaria la inversión en infraestructura para fortalecer la enseñanza de las ciencias. Sin embargo, están de acuerdo en que se necesita mayor equidad en la cantidad de estudiantes que se tiene en el aula y en el uso de computador en la institución, ratificando la respuesta de los estudiantes que no se hace uso de las TIC para la enseñanza de las ciencias.

Tabla 8

Factores relacionados a la Categoría Escuela

\begin{tabular}{|c|l|}
\hline N & \multicolumn{1}{|c|}{ Factor } \\
\hline 1 & Diferencias entre los estudiantes de la misma escuela. \\
\hline 2 & Análisis en los resultados de pruebas internas y externas. \\
\hline 3 & Marcada influencia de la zona donde se ubica la escuela. \\
\hline 4 & Influencia de la violencia propia del contexto. \\
\hline 5 & Desigualdad en el número de estudiantes en las aulas. \\
\hline 6 & Tenencia de texto y cuadernos en los estudiantes. \\
\hline 7 & Falta de equidad en el uso del computador. \\
\hline 8 & Oportunidades de uso del computador fuera de la escuela. \\
\hline 9 & Inversión en reformas en las escuelas. \\
\hline 10 & Desigualdad en la calidad de educación que ofrecen las diferentes instituciones educativas. \\
\hline 11 & Tiempo en la enseñanza de las ciencias. \\
\hline 12 & Compromiso por parte del estudiante frente al área. \\
\hline 13 & Ausencia de oportunidades para formación y capacitación en las áreas. \\
\hline
\end{tabular}

Fuente: Elaboración propia 
Gráfica 7

Resultado en porcentaje de los

factores de la Categoría Escuela

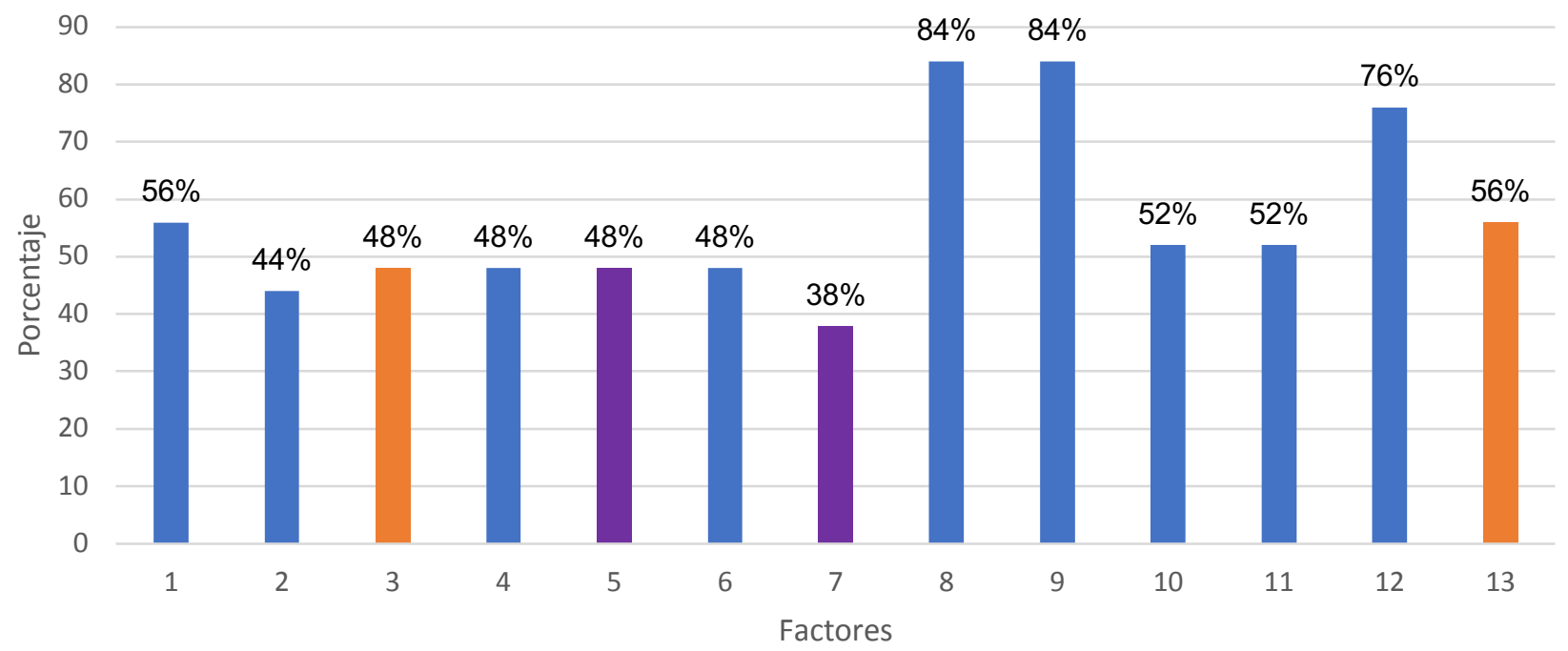

Fuente: Elaboración propia

\section{Segunda etapa: Análisis de significación bivariado}

Inicialmente, es importante señalar que no se encontraron diferencias en las frecuencias sexo $(\chi 2(3, N=795)$ $=1.10, p>0.05)$ y edad $(\chi 2(18, N=795)=14.27, p>0.05)$ que influyeran o estuvieran asociadas al resultado de las competencias. Por tal razón, estas dos variables no se han tenido en cuenta para fortalecer la investigación realizada.

Así mismo, los resultados obtenidos en los tres cuestionarios y para comprenderlos, se buscaba conocer el grado de asociación individual de las variables, para ello se usó la prueba de Chi-cuadrado ( $\chi 2$ ), de esta manera, si existe significación menor ó igual a 0.05 se rechazó la hipótesis de independencia y permite corroborar la asociación de las variables. Al encontrar asociación, se busca identificar su fuerza a través del estadístico V de Cramer, entonces, su resultado puede estar en el rango $0-1$, de tal manera, que los valores cercanos a 0 indican no asociación y los próximos a 1 fuerte asociación (Pardo \& Ruiz, 2001).

De acuerdo con las características mencionadas en el párrafo anterior, después de aplicar la prueba de Chicuadrado (x2) y determinar que la significación era menor ó igual a 0.05 con todos los factores incluidos en las cuatro categorías, se empleó el estadístico $V$ de Cramer para determinar la fuerza de la asociación con los factores que la presentaron. Para ello, en la tabla №9 se relacionan las categorías, descriptores y factores que presentaron dicha asociación. 


\section{Tabla 9}

Indicadores que representan asociación según la prueba de Chi-cuadrado con $\mathrm{P}<0.05$

\begin{tabular}{|l|l|l|l|}
\hline Categoría /Descriptor & Factores & gl & X2XX2 \\
\hline Familia/ Antecedentes Escolares & Presenta justificación cuando falta a clase. & 6 & 18.79 \\
\hline $\begin{array}{l}\text { Familia/Participación de padres en actividades } \\
\text { escolares }\end{array}$ & $\begin{array}{l}\text { Supervisión en actividades por parte de los } \\
\text { padres de familia }\end{array}$ & 9 & 17.96 \\
\hline $\begin{array}{l}\text { Contexto socioeconómico/Indicadores de } \\
\text { pobreza y desigualdad }\end{array}$ & $\begin{array}{l}\text { Desigualdad en los ingresos económicos y bienes } \\
\text { que poseen en el hogar. Tener o no tener tablet }\end{array}$ & 6 & 15.21 \\
\hline $\begin{array}{l}\text { Contexto socioeconómico/equilibrio en el } \\
\text { desarrollo Humano }\end{array}$ & $\begin{array}{l}\text { Tasa de analfabetismo de primaria en los } \\
\text { adultos del hogar }\end{array}$ & 6 & 14.60 \\
\hline $\begin{array}{l}\text { Contexto socioeconómico/equilibrio en el } \\
\text { desarrollo Humano }\end{array}$ & $\begin{array}{l}\text { Falta de educación secundaria en los adultos del } \\
\text { hogar }\end{array}$ & 6 & 17.23 \\
\hline $\begin{array}{l}\text { Escuela/Recursos de la escuela } \\
\text { Oportunidades de facilidad de acceso a } \\
\text { información virtual fuera de la escuela }\end{array}$ & 2 & 7.59 \\
\hline Escuela/Recursos de la escuela & Inversión en reformas en las escuelas & 2 & 4.04 \\
\hline $\begin{array}{l}\text { Enseñanza de las ciencias /Proceso de } \\
\text { enseñanza de las ciencias }\end{array}$ & Interés por las TIC en la clase & 24.53 \\
\hline $\begin{array}{l}\text { Enseñanza de las ciencias /Actitud por parte del } \\
\text { docente }\end{array}$ & Indisciplina en clase & 6 & 25.66 \\
\hline $\begin{array}{l}\text { Enseñanza de las ciencias /Actitud por parte del } \\
\text { docente }\end{array}$ & Importancia en las tareas dejadas en clase & 9 & 26.39 \\
\hline
\end{tabular}

Fuente: Elaboración propia

\section{Tercera etapa: Modelos de las categorías}

Después de identificar la asociación las variables dependientes, se incluyeron en un modelo de regresión logística multinominal o multivariante, el cual permite identificar las probabilidades de registro de los desempeños. Entonces, se evaluaron los factores de manera simultánea según resultados de la prueba Chi-cuadrado (X2), con la variable independiente "desempeños", identificando su efecto de forma ajustada (exponencial de B). De acuerdo con los resultados de la prueba de competencias científicas, donde los estudiantes se ubicaron en desempeño bajo, este resultado fue tomado como categoría de referencia en las regresiones logísticas multinominales. Es importante señalar que, aunque estadísticamente los resultados: "no registra" fueran significativos, no fueron interpretados porque son imprácticos. La tabla N 10 relaciona las categorías, descriptores y factores con las probabilidades de obtener un mejor desempeño si se tienen en cuenta estos factores en los procesos que se llevan en la enseñanza y aprendizaje de las ciencias. 
Tabla 10

Tabla de probabilidades y desempeños

a obtener por indicador

\begin{tabular}{|l|l|l|l|}
\hline \multicolumn{1}{|c|}{ Categoría /Descriptor } & \multicolumn{1}{|c|}{ Factores } & Probabilidad & \multicolumn{1}{|c|}{$\begin{array}{c}\text { Desempeño a } \\
\text { obtener }\end{array}$} \\
\hline Familia/ Antecedentes Escolares & Presenta justificación cuando falta a clase. & $4-8$ veces & Alto y básico \\
\hline $\begin{array}{l}\text { Familia/Participación de padres } \\
\text { en actividades escolares }\end{array}$ & $\begin{array}{l}\text { Supervisión en actividades por parte de los } \\
\text { padres de familia }\end{array}$ & 2 veces & Básico \\
\hline $\begin{array}{l}\text { Contexto } \\
\text { socioeconómico/Indicadores de } \\
\text { pobreza y desigualdad }\end{array}$ & $\begin{array}{l}\text { Desigualdad en los ingresos económicos y } \\
\text { bienes que poseen en el hogar. Tener o no } \\
\text { tener tablet }\end{array}$ & 6 -13 veces & Alto y básico \\
\hline $\begin{array}{l}\text { Contexto } \\
\text { socioeconómico/equilibrio en el } \\
\text { desarrollo Humano }\end{array}$ & $\begin{array}{l}\text { Tasa de analfabetismo de primaria en los } \\
\text { adultos del hogar }\end{array}$ & 4 veces & Básico \\
\hline $\begin{array}{l}\text { Escuela/Recursos de la escuela } \\
\text { Enseñanza de las } \\
\text { ciencias/Proceso de enseñanza } \\
\text { de las ciencias }\end{array}$ & Inversión en reformas en las escuelas & Menor \\
\hline $\begin{array}{l}\text { Enseñanza de las ciencias } \\
\text { IActitud por parte del docente }\end{array}$ & Indisciplina en clase & $5-7$ veces & Básico \\
\hline $\begin{array}{l}\text { Enseñanza de las ciencias } \\
\text { /Actitud por parte del docente }\end{array}$ & Importancia en las tareas dejadas en clase & básico \\
\hline
\end{tabular}

Fuente: Elaboración propia

\section{Cuarta etapa: Asociación general de puntaje y factores}

Para fortalecer el estudio realizado, se llevó a cabo un análisis de componentes principales, el cual permitió la reducción de los factores. De esta manera, al excluir los datos que incluían la categoría no registra, se concentraron en 16 ejes, donde el uno y dos explican el $19.21 \%$ de la inercia total de la asociación entre las categorías.

\section{Conclusiones}

Los procesos de enseñanza y aprendizaje de las ciencias naturales desde las primeras edades llevan consigo el desarrollo y fortalecimiento de las competencias científicas, en concordancia, Macedo \& Neida (1997) señalan que la enseñanza de las ciencias favorece en niños y niñas el desarrollo de la observación, análisis, razonamiento, comunicación y abstracción, de esta manera estos procesos de pensamiento dan la posibilidad de que los estudiantes construyan su conocimiento de manera autónoma, así mismo, se forjan su propia cultura científica. Por ello, las ciencias naturales deben aproximar al estudiante al mundo de la vida (MEN, 1998), fortaleciendo los presaberes que traen desde su entorno y transformándolos en conceptos más elaborados para dar validez científica.

En concordancia con Chamizo \& Pérez (2017) “la enseñanza de las ciencias requiere que los estudiantes no sólo construyan conocimientos disciplinares, sino que también sean capaces de comprender cómo se han obtenido, para de esta manera puedan experimentarlos y aplicarlos de acuerdo con su contexto de vida". Es decir, al evaluar las competencias científicas, desde el uso del conocimiento científico, la explicación de fenómenos y la indagación, se reconoce la validez de los procesos educativos que se desarrollan en el aula. Los cuestionarios dos y tres llamados "factores asociados" permitieron reconocer en estudiantes y docentes la implicación que 
tienen elementos del aula, la escuela, la familia y el contexto en el aprendizaje de las ciencias, específicamente en el desarrollo de competencias científicas.

Así mismo, de acuerdo con Barahona et al. (2018) el éxito académico de los estudiantes es multifactorial, por tal razón, el aprendizaje no depende solo del trabajo en el aula, factores como la familia y el contexto determinan el interés y la motivación por adquirir nuevos conocimientos y profundizar en diferentes áreas como proyecto de vida.

Desde la categoría familia se observa que la supervisión de actividades por parte de los padres de familia y la formación integral de niños y niñas, evidenciada en la responsabilidad de entregar excusa cuando no se asiste a clase presenta mayor probabilidad de obtener mejores resultados en las pruebas de competencias científicas. Al respecto, Sánchez-Martínez \& Ricoy (2018) señalan que "la familia y la escuela, como instituciones milenarias de peso, continúan siendo ámbitos esenciales de influencia para el desarrollo y la educación de los más pequeños". Así mismo, desde los resultados se observa que la familia es considerada como el primer agente de socialización y contribuye al desarrollo físico, emocional, intelectual y social de los más pequeños. En consonancia, Carvajal \& Castillo (2018) concluyen que la participación y apoyo familiar en los procesos educativos poseen alta influencia en el comportamiento de otras variables predominantes en el proceso educativo.

La categoría contexto socioeconómico señala que, de los factores consultados, reducir la tasa de analfabetismo en el hogar y mantener equidad en los recursos que sirven de apoyo al proceso de aprendizaje pueden generar mayores probabilidades de obtener mejores resultados en la prueba de competencias científicas. De esta manera, se puede evidenciar que el contexto incide directamente en lo que hace y logra la escuela, en contraste con estudios realizados al identificar inequidades socioeconómicas, estas se reflejan en los logros de aprendizaje Villalta \& Assael (2018).

Desde la categoría Escuela se evidencia que la inversión en infraestructura en las escuelas puede ampliar las probabilidades de obtener mejores resultados en las pruebas de competencias científicas, visto de esta manera, el mejoramiento de laboratorios y las salas de informática, especialmente, aquellos elementos que ofrecen mayores probabilidades de fortalecer la construcción de conocimientos en experimentación y, con ello, explorar fenómenos físicos y químicos.

La última categoría corresponde a la enseñanza de las ciencias naturales, siendo esta fundamental en el desarrollo de competencias científicas. En este sentido, factores como: la convivencia en el desarrollo de la clase, el uso de las TIC y el valor que se le da a la tarea, pueden influir positivamente en los resultados asociados al desempeño en competencias científicas. El principal actor de este proceso es el docente, quien se debe preocupar por construir un proceso adecuado y apto para cada población de estudiantes que atienda.

De acuerdo con lo anterior se puede observar tres posturas que se presentan en la investigación y se soportan sobre sus respectivos refrentes teóricos: La primera en palabras de Martín, Prieto \& Lupión (2014): los docentes deben tener en cuenta en su práctica los cambios sociales que se producen, los cuales requieren de cambios educativos a desarrollar, entonces, estos cambios se convierten en espacios creadores de conocimiento y capacitadores de futuros ciudadanos y profesionales. Así que el hecho de enseñar ciencias no solo consiste en ingresar a un aula y explicar una serie de conocimientos.

La segunda y de acuerdo con Buitrago (2020) el aprendizaje debe centrarse en el alumno, en el desarrollo de habilidades y competencias, en los resultados e incluso se han asociado al éxito escolar y académico, sin embargo, el docente y su función de enseñanza se ve desligada del proceso, en ocasiones cuando los resultados son positivos es relegada y cuando los resultados son negativos es desprestigiada. La tercera y de acuerdo con Coronado \& Arteta (2015) la enseñanza de las ciencias naturales y el desarrollo de las "competencias científicas en los estudiantes, obedece en gran medida al conocimiento profesional del docente, especialmente al conocimiento didáctico del contenido, CDC, el cual se fundamenta en otros tipos de conocimientos". 


\section{Referencias bibliográficas}

Barahona, P., Veres, E. \& Barahona, M. (2018). Factores asociados a la calidad de la educación en Chile. Revista Internacional de Investigación en Ciencias Sociales. 14. 17-30. Recuperado de http://revistacientifica.uaa.edu.py/index.php/riics/article/view/482

Buitrago, R. (2020). El aprendizaje, la enseñanza, los pensamientos y las interacciones en la escuela. Praxis \& Saber. 25. 9-20. https://doi.org/10.19053/22160159.v11.n25.2020.10580

Carvajal, C. \& Castillo, C. (2018). Estudio metodológico de las variables que componen el constructo de "apoyo y participación de la familia en los procesos educativos". Estudios Pedagógicos XLIV. 115-133. http://revistas.uach.cl/index.php/estped/article/view/3601

Chamizo J. \& Pérez Y. (2017). Sobre la enseñanza de las ciencias naturales. Revista iberoamericana de educación. 74. 23-40. Recuperado de https://doi.org/10.35362/rie741624

CLAVIJO, C. D. (2018). Competencias del docente universitario en el siglo XXI. Revista Espacios 39(20), 22. Recuperado de: https://www.revistaespacios.com/a18v39n20/a18v39n20p22.pdf

Coronado, M \& Arteta, J. (2015). Competencias científicas que propician docentes de Ciencias naturales. Zona próxima, 23, 131-144. Recuperado de http://dx.doi.org/10.14482/zp.22.5832

Hernández Sampieri, R., Fernández Collado, C., \& Baptista Lucio, P. (2014). Metodología de la investigación: Roberto Hernández Sampieri, Carlos Fernández Collado y Pilar Baptista Lucio (6a. ed. --.). México D.F.: McGraw-Hill. Págs. 563-576

Icfes (2016). Instituto colombiano para el fomento de la educación superior. Guía de Interpretación y Uso de Resultados de las pruebas Saber $3^{\circ}, 5^{\circ}$ y $9^{\circ}$. Pág. 5. Bogotá-Colombia.

Macedo B. \& Neda J (1997). Un Currículo Científico para Estudiantes de 11 a 14 años. OEI - UNESCO/Santiago. Organización de Estados Iberoamericanos para la Educación, la Ciencia y la Cultura. Santiago-Chile.

Martín, C, Prieto, T. \& Lupión, T. (2014). Profesorado de ciencias en formación inicial ante la enseñanza y el aprendizaje de las ciencias: ¿perfil innovador o tradicional?. Revista Electrónica Interuniversitaria de Formación del Profesorado, 17 (1), 149-163. DOI: http://dx.doi.org/10.6018/reifop.17.1.180811

MEN (1998). Lineamientos curriculares en Ciencias Naturales y Educación Ambiental. Ministerio de Educación Nacional. Bogotá-Colombia.

Pardo, A., \& Ruiz, M. (2001). Guía para el análisis de datos. En SPSS 10.0. Madrid: UNAM Ediciones.

R Core Team. (2018). R: A Language and Environment for Statistical Computing. Vienna, Austria: R Foundation for Statistical Computing. Recuperado de https://www.R-project.org/

Sánchez-Martínez \& Ricoy M. (2018) Posicionamiento de la familia ante el uso de la tableta en el aprendizaje del alumnado de Educación Primaria. Digital Education Review. 33.267.283. Recuperado de http://greav.ub.edu/der/

UNESCO (2015). Informe de resultados TERCE. Informe de resultados tercer estudio regional comparativo y explicativo. Factores Asociados. Pág. 7.

Villalta, M. \& Assael, C. (2018). Contexto socioeconómico, práctica pedagógica y aprendizaje autónomo en el aula. Recuperado de Estudios Pedagógicos XLIV. 49-68.

https://revistaschilenas.uchile.cl/handle/2250/86302

Vivanco, M. (2005). Muestreo estadístico. Diseño y Aplicaciones. (Editorial Universitaria). Santiago de Chile. ISBN. 956-11-1803-3 